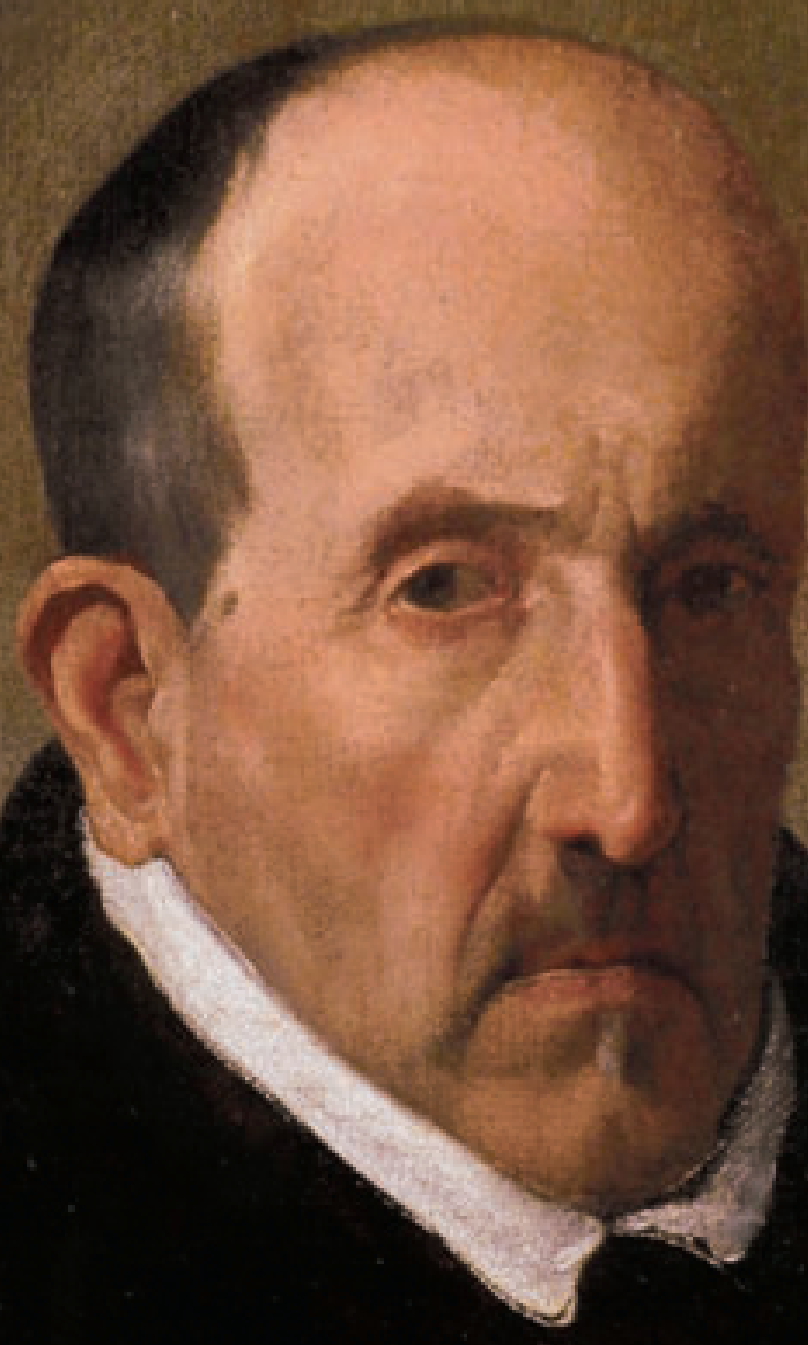




\section{La tecnología unida a la restauración para el estudio de Retrato del poeta D. Luis de Góngora y Argote}

Lourdes Núñez Casares, Lourdes Martín Garcia, Gabriel Ferreras Romero, Auxiliadora Gómez Morón, Centros de Intervención e

Investigación y Análisis del IAPH. Jacques Castaing, Adrián Durán Benito, Centre de Recherche et de Restauration des Musées de France CNRS. Ángel Jesús Polvorinos del Río, Dpto. de Cristalografía, Mineralogía y Quimica Agrícola de la U. de Sevilla

\section{Resumen}

El presente artículo hace referencia a la pintura al óleo sobre lienzo denominada Retrato del poeta D. Luis de Góngora y Argote, perteneciente a la Fundación Lázaro Galdiano de Madrid. El 7 de febrero de 2008 llegó a las dependencias del IAPH tras formar parte de la muestra expositiva Teatro de Grandezas (Andalucia Barroca), organizada por la Junta de Andalucía en el Hospital Real de Granada.

Esta obra es una de las cuatro versiones más importantes que se encuentran en el mundo sobre Góngora, de las cuales los expertos no han dilucidado aún si ésta puede considerarse original, réplica o copia de gran calidad artística.

Este lienzo es crucial en la vida de Velázquez, ya que le abre las puertas de la Corte al ver ésta el gran estudio psicológico que realiza del personaje y como consecuencia cambia no sólo el estatus del pintor, sino también los temas que representa centrándose en los retratos reales.

Gracias a la restauración se ha recuperado la calidad de la obra y se han podido estudiar con profundidad la composición material y la técnica empleada por el autor, abriendo un camino para que continúen los estudios sobre la misma.

\section{Palabras clave}

Análisis / Centro de Intervención / Fundación Lázaro Galdiano / Góngora, Luis de / Intervención / Instituto Andaluz del Patrimonio Histórico / Investigación / Lienzo / Limpieza / Óleo / Pinturas / Reintegración / Retratos / Velázquez, Diego / 1622 


\section{ANÁLISIS HISTORIOGRÁFICO Y ESTADO DE LA CUESTIÓN}

Se sabe que Francisco Pacheco, en su tratado Arte de la Pintura, I. cap. VIII, escribió: "Hizo, a instancia mía, un retrato de don Luis de Góngora, que fue muy celebrado en Madrid, y por entonces no hubo lugar de retratar los Reyes, aunque se procuró", refiriéndose al retrato que le encargó a su joven yerno Diego Velázquez, cuando en 1622, realiza su primer viaje a Madrid.

El encargo debió de tener dos intenciones claras: una, la de obtener la imagen real del célebre poeta cordobés don Luis de Góngora y Argote (1561-1627), a quien quizás Pacheco no conociera directamente, que tendría la edad de sesenta o sesenta y un años de edad y estaba en la cumbre de su fama. Así mismo, obtendría el tratadista la imagen del gran poeta creador del culteranismo con la idea de incluirla en su Libro de descripción de verdaderos retratos de ilustres y memorables varones, fechado en 1599 en Sevilla (aunque esta obra no se concluyera nunca y fuera destinada a grabados que tampoco se llegaron a terminar). Y quizás, la segunda intención con su encargo podría ser dar a conocer al joven Velázquez, para que contactara con personajes importantes de la Corte, que a su vez, le introdujeran en ese círculo, ya que Góngora era en esos años capellán de honor del joven rey Felipe IV y figura de relevancia por su poemas escritos, pues al poco tiempo, al año siguiente, lo requiere el conde-duque de Olivares para que volviera a Madrid pintando después, en el verano de 1623, al propio rey.

Dicha obra fue muy conocida y admirada en Madrid, como consta a través de Palomino, haciéndose varias réplicas o copias que demuestran su éxito y fama.

En la actualidad no hay unanimidad por parte de los historiadores en establecer qué versión fue la original. Existen cuatro versiones significativas del retrato: la conservada en el Museo de Boston, ésta de la Fundación Lázaro Galdiano, otra en el Museo del Prado y la que se conserva en la actualidad en una colección particular de San Sebastián.

La que se viene considerando generalmente y casi por unanimidad como la obra original es la del Museum of Fine Arts de Boston (ver imagen 1), que siempre ha sido la más alabada por la crítica especializada, desde la década de los años veinte del siglo pasado, cuando el hispanista Mayer (1921: 74) la conoce y sabe que perteneció a la colección del marqués de la Vega Inclán, y que en 1931 se expuso en Londres en la muestra celebrada por la Casa de Thomás Harris Limited sobre Pintores Españoles; posteriormente, en 1932, la adquiere el Museo de Boston, donde en la actualidad se conserva (ALLENDE-SALAZAR, 1925; TRAPIER, 1948; ASTURIASBARDI, 1970; BROWN, 1986, etc.).

Este retrato tuvo una corona de laurel sobrepintada, visible en las radiografías, que posteriormente se eliminó (GARRIDO PÉREZ, 1992), como los retratos laureados de los demás poetas (Gutierre de Cetina, Fernando de Herrera, Rodrigo Caro, Baltasar de Alcázar, Francisco Quevedo, Cristóbal Moxquera, etc.) que Pacheco dibujó para su Libro de descripción de verdaderos retratos de ilustres y memorables varones.

La crítica especializada considera y reconoce este retrato de Boston como el prototipo pintado con la técnica en otras obras juveniles del maestro, con la materia "de la cabeza tratada con planos autónomos, como facetada"; Camón Aznar en 1964 lo niega y además reprocha, considerándola copia posterior a la conservada en la Fundación Lázaro, pues la considera "desenfadada y de impetuoso barroquismo", por lo tanto posterior a su primera etapa. Pero será precisamente estas matizaciones técnicas de lo que se valdrá el profesor Julián Gállego (1991: 112) para subrayar que es lo que le da mayor valor a ese ejemplar y está más conforme con el "modelado riguroso, duro" de sus primeras obras pintadas en Sevilla, como definió antes Pantorba en 1955.

Del retrato de don Luis de Góngora de la Fundación Lázaro Galdiano, que se ha restaurado recientemente en el IAPH, se conoce que fue adquirido por el coleccionista don José Lázaro (1862-1947), entre los años 1912 y 1913, a través del anticuario Miguel Borondo, que poseía un pequeño comercio de antigüedades en la plaza de Isabel II, cercano al Teatro Real de Madrid'.

Esta versión ardientemente es defendida por el primer conservador de dicha Fundación, José Camón Aznar (1964), como la obra original de Velázquez, ya que supuso que pudiera ser el retrato de Góngora que se recoge en el inventario de los bienes del propio Velázquez a su muerte en 1660 y que fue heredado a su vez de su suegro Francisco Pacheco.

Así mismo, Camón Aznar consideraba que este lienzo procedia, desde 1677, de la colección del marqués del Carpio y en los sucesivos inventarios de la misma, se registra un retrato del poeta atribuido a Velázquez, que tal vez pudiera ser este mismo retrato.

Además, Camón Aznar técnicamente dice que "la capa de color es delgada, pero todas las fluencias del modelo se tratan con el más matizado claroscuro. Se encuentra realizado el cuadro en ese feliz momento en que el pintor se ha liberado de la plasticidad tenebrista de su etapa sevillana y todos los relieves de este rostro, aún tratado con un modelado vigoroso, se hallan dulcificados por una blandura de pincel, por una entonación aquilatada, que permiten el realismo tan apuntado de esta faz, cuya piel exangüe transparenta una salud tarada y un espíritu aguileño e incisivo".

Y consideró siempre réplica posterior al retrato del Museo de Boston.

El retrato del Museo del Prado (no 1 223) (imagen 2), hoy considerado por la crítica en general como una buena copia del taller del propio Velázquez (GARRIDO PÉREZ, 1992), fue durante mucho tiempo la más estimada por su emplazamiento. Sin 


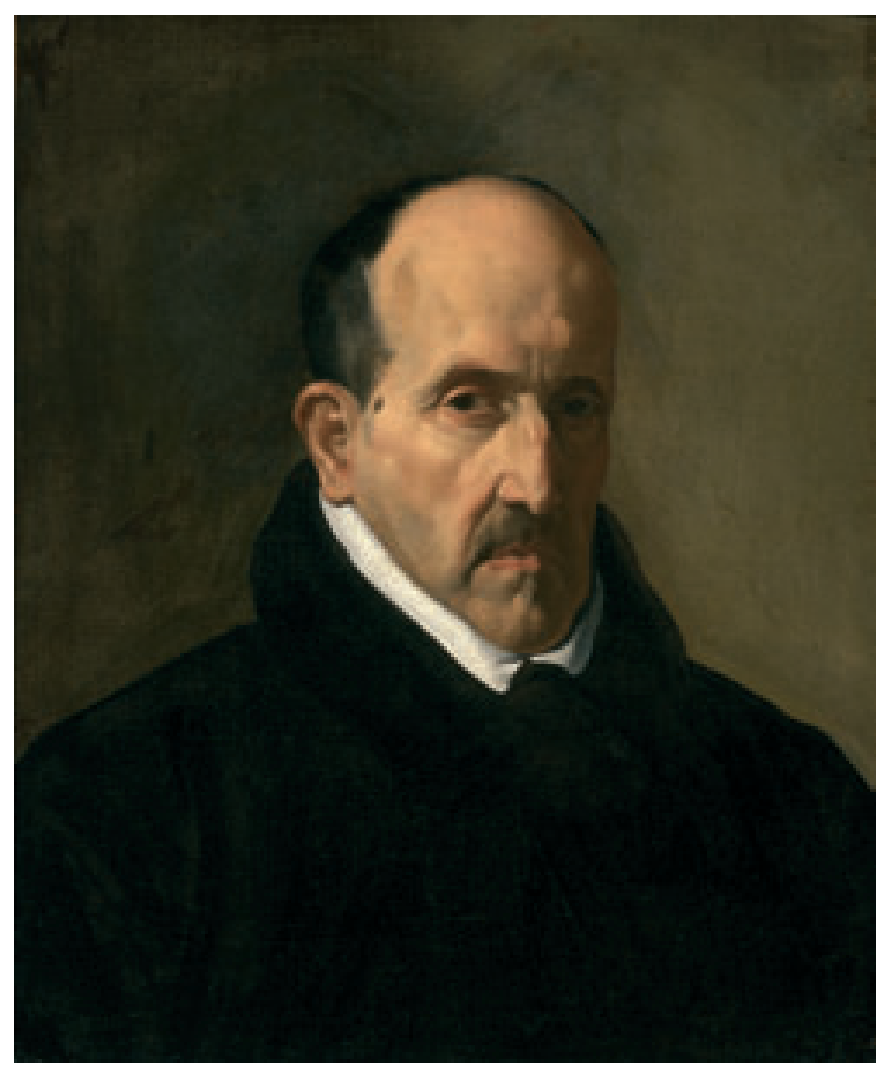

1. Versión de la obra conservada en el Museum of Fine Arts de Boston. Fuente: Photograph (C), noviembre 2009, Museum of Fine Arts, Boston

embargo, el cuadro del Prado es hoy claramente una obra más pobre y sin la sutil vibración de los ejemplares de Boston y de la Fundación Lázaro.

La cuarta versión del retrato de Góngora se encontraba en la antigua colección de Ramón Aras Jáuregui, en Bilbao; actualmente el cuadro está en San Sebastián². Restaurado en 1910 y muy recortado hasta dejar sólo la cabeza, procede de la colección GandariIlas, como recogió por varios datos Pantorba (1955) que lo llegó a considerar incluso como original.

También citar otro quinto retrato de Góngora, pero menos importante, el que perteneció al marqués de Cabriñana, Don Ignacio María de Argote, que lo donó al Museo Iconográfico y posteriormente pasó al Museo del Prado, siendo depositado en la Academia de la Historia, donde se conserva en la actualidad.

Esta última versión, de mayores dimensiones, posee la curiosidad de que incluye brazos y manos que sostienen un bonete de sacerdote. Como el donante era descendiente de la familia cordobesa del retratado (Argote), es posible que el lienzo sea derivación directa de un original. Es un cuadro que sólo lo ha tenido en cuenta el profesor Pérez Sánchez (2005), pero es indudable que se trata de una copia lejana del maestro.

Lo que parece estar claro es recordar de nuevo las palabras del propio Pacheco que dice textualmente que Velázquez "lo hizo a instancia mía, un retrato de don Luis de Góngora que fue muy celebrado en Madrid", lo que parece asegurar que el retrato se

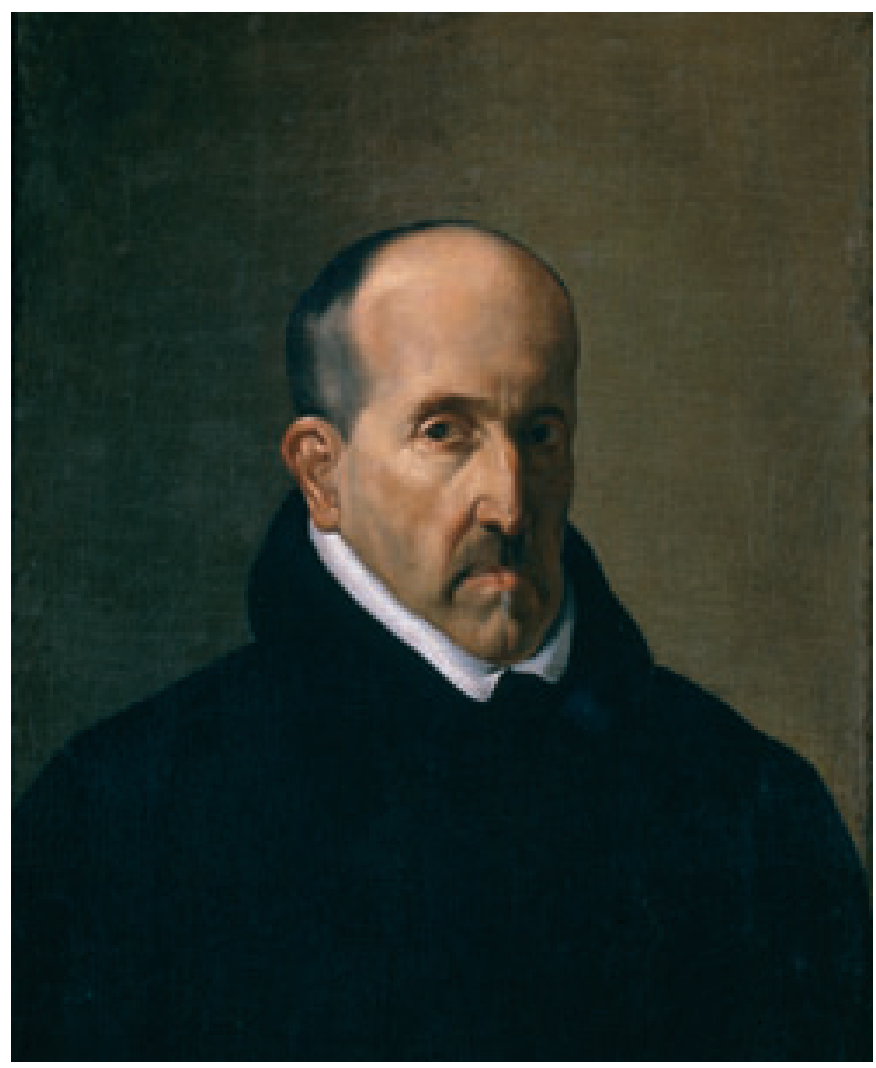

2. Versión de la obra conservada en el Museo del Prado. Fuente: (C) Museo del Prado-Madrid. España

hizo para servir de modelo en el citado libro, que como es sabido, se nutrió de retratos ajenos y usó siempre de la corona de laurel para honrar a los poetas.

El profesor Pérez Sánchez (2005) considera la versión del retrato de don Luis de Góngora de la Fundación Lázaro Galdiano como "desde luego una obra más suave y matizada, y el color, delicado y transparente, muestra una considerable maestría que quizás permita considerarla como réplica del propio pintor, algo más tardia". Elías Tormo (1913), que no conoció el lienzo de Boston, lo creía original, y Gudiol (1973) lo cree "réplica que sólo Velázquez pudo realizar" dándole precedencia al de Boston como original. Sin embargo, Garrido (1992: 149) considera la versión de Boston temprana para la fecha de 1622, por la forma en que está pintada y por los materiales que en ella se utilizan en comparación con otras pinturas analizadas.

\section{ESTUDIOS CIENTÍFICOS APLICADOS A LA OBRA}

El análisis en profundidad de los materiales y la técnica empleada en la elaboración de esta relevante obra ha requerido el uso de métodos complementarios entre sí. Algunos de ellos se caracterizan por requerir la toma de micromuestras para su realización mientras que en otros no es necesario. En el primer caso, con el fin de no ocasionar ningún daño a la obra, la extracción de muestras se ha llevado a cabo en los bordes del lienzo una vez desmontado el marco de la pintura. Se han utilizado diversas técnicas analíticas, desde el estudio estratigráfico 
hasta técnicas instrumentales más complejas como la cromatografía de gases acoplada a espectrometría de masas (GC/MS) o la microscopia electrónica de barrido, técnicas que ofrecen una amplia información sobre gran variedad de materiales tanto de naturaleza orgánica como inorgánica. Asimismo se han utilizado en este estudio técnicas no destructivas (que no requieren la extracción de muestras, como la difracción de rayos $X$ y la fluorescencia portátil), en particular para el análisis de zonas delicadas del cuadro como el rostro o las vestimentas donde no era posible la toma de muestras.

La aplicación de estas innovadoras técnicas no invasivas ha sido posible a través de la colaboración con el laboratorio de investigación del Centre de Recherche et de Restauration des Musées de France (C2RMF) y la Universidad de Sevilla, en el marco del proyecto europeo EU ARTECH para la aplicación de nuevas tecnologías al ámbito patrimonial. En este proyecto se ha desarrollado un equipo portátil (imagen 3) que combina difracción (DRX) y fluorescencia de rayos $X$ (FRX) en el mismo aparato (GIANNONCELLI et ál., 2008; DURÁN et ál., 2009), lo que permite realizar in situ y de forma no invasiva el análisis químico elemental y estructural de los compuestos analizados.

La combinación de los resultados obtenidos con ambos tipos de técnicas ha permitido sacar interesantes conclusiones que han aportado una información imprescindible tanto para abordar la restauración de la obra como para su investigación histórica.

Para el estudio al microscopio óptico las muestras han sido incluidas en una resina de metacrilato y cortadas en secciones transversales que posteriormente se han pulido. En ellas se ha estudiado la superposición de capas pictóricas, su espesor, homogeneidad y apariencia detectándose asimismo la presencia de repintes. De todo ello se ha obtenido información sobre los pigmentos empleados así como sobre la técnica pictórica. También ha permitido conocer el estado de los materiales y las posibles restauraciones que ha sufrido la obra a lo largo de su historia.

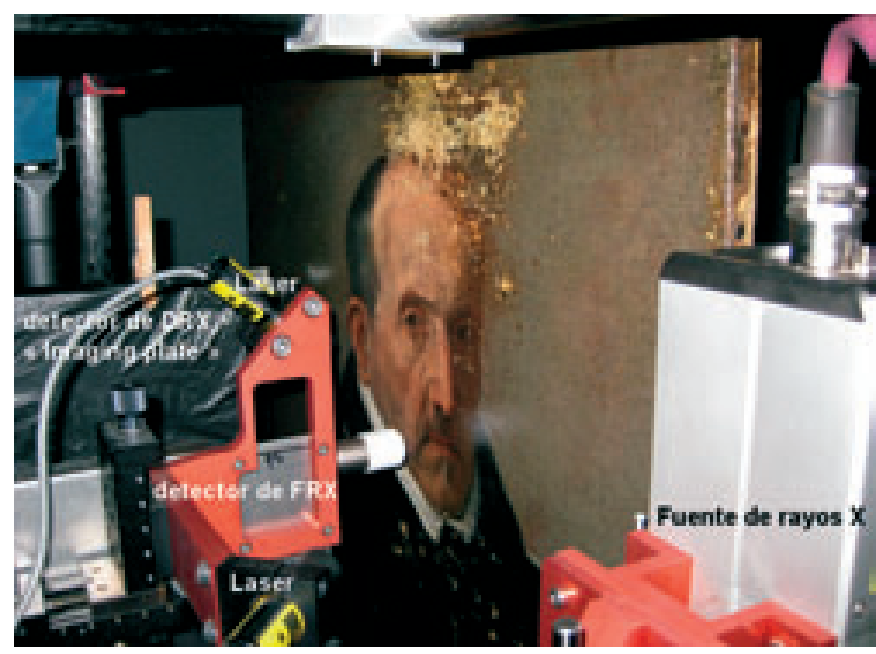

3. Equipo de difracción y fluorescencia de rayos $X$ portátil diseñado y construido en el Centre de Recherche et de Restauration des Musées de France.

Foto: Jacques Castaing
Los pigmentos y cargas inorgánicas se han analizado por medio de la microscopía óptica, completándose el estudio con el microscopio electrónico de barrido por energía dispersiva de rayos $X$ (SEM-EDX). También se han empleado técnicas no invasivas de fluorescencia de rayos $X(F R X)$ y difracción (DRX) para el análisis de las zonas donde era imposible la toma de muestras. Estas técnicas permiten realizar análisis simultáneos y determinar en el mismo punto la composición química elemental y mineralógica, información imprescindible para un estudio global de los materiales de la obra.

La fuente de rayos $X$ está formada por un tubo iMOXS-MFR (IFG, Adhershof, Berlin, Alemania) con ánodo de cobre que trabaja a una potencia máxima de $30 \mathrm{~W}$, voltaje de $40 \mathrm{kV}$ e intensidad de $700 \mu \mathrm{A}$, y está equipada con unas lentes policapilarios y filtro de niquel de $15 \mu \mathrm{m}$. La medida de los rayos $X$ de fluorescencia se realiza con un detector Silicon Drift (Röntec GMBH) refrigerado por efecto Peltier a $-10^{\circ} \mathrm{C}$, teniendo una resolución de $150 \mathrm{eV}$ a $5.9 \mathrm{keV}$. La adquisición de cada espectro se realizó durante 300 segundos. El detector empleado para la difracción de rayos $X$ consiste en imaging plates, que son detectores bidimensionales con dimensiones $15 \times 30 \mathrm{~cm}$, que permiten recoger los anillos de difracción procedentes de la muestra analizada; la recogida de datos se produce en unos 20 minutos, posteriormente el imaging plate es leído mediante un escáner y la imagen transformada en un diagrama de difracción de rayos $X$ convencional con la ayuda del programa FIT2D.

El reducido tamaño y peso del equipo ha facilitado la realización de los estudios en los propios talleres de restauración del IAPH sin necesidad de desplazar la obra a otras instalaciones. El sistema está provisto de mecanismos de movimiento en las tres direcciones del espacio $(x-y-z)$ mediante tornillos micrométricos que permiten el análisis de cualquier punto de la obra.

Una limitación de este tipo de estudios no invasivos es conocer la correspondencia de los compuestos que se identifican con las distintas capas de la estratigrafía. Para ello es importante determinar el poder de penetración de los rayos $X$ que define la profundidad de la zona analizada dentro del material objeto de estudio. Teniendo en cuenta que el ángulo de incidencia de los rayos $X$ es de aproximadamente $10^{\circ}$, podemos estudiar hasta una profundidad de $25 \mu \mathrm{m}$ en el caso de materiales constituidos por elementos ligeros $(\mathrm{Al}, \mathrm{Si}, \mathrm{K})$ y de $10 \mu \mathrm{m}$ para materiales pesados $(\mathrm{Pb}, \mathrm{Hg}, \mathrm{Sn})$.

Para los estudios por FRX, la profundidad del análisis depende de la naturaleza de los elementos químicos implicados ( $\mathrm{Al}$, $\mathrm{Si}, \mathrm{K}, \ldots, \mathrm{Pb}, \mathrm{Hg}, \mathrm{Sn}$ ) y además de la energía de los rayos $X$ de fluorescencia usada para la detección de los elementos. La profundidad de la zona analizada puede variar entre $1 \mu \mathrm{m}$ para el caso del silicio hasta llegar a valores superiores a $150 \mu \mathrm{m}$ para elementos como el estaño en una matriz de hematite $\left(\mathrm{Fe}_{2} \mathrm{O}_{3}\right)$ (GIANNONCELLI et ál., 2008). 
En el caso concreto de esta obra se ha identificado un alto contenido de blanco de plomo en las capas pictóricas. Este compuesto posee un elevado poder de absorción de los rayos $X_{1}$ lo que provoca que el análisis químico elemental y mineralógico se limite a las capas más superficiales.

Los aglutinantes se han estudiado al microscopio óptico mediante técnicas de tinciones selectivas sobre las secciones trasversales para su localización y para la determinación de la naturaleza química del aglutinante, completándose el estudio mediante cromatografía de gases acoplada a espectrometría de masas (GC/MS). Esta última técnica permite la separación e identificación de los constituyentes característicos e identificar los aglutinantes orgánicos y ha sido también la empleada para la determinación de ceras y resinas.

Las muestras se han tratado con el reactivo de metilación Methprep II en el caso de sustancias de tipo cera u óleo-resinosas. Los aminoácidos, junto también a los ácidos grasos, se analizaron mediante cromatografía de gases a través de derivatización con TBDMSTFA en piridina.

El estudio de compuestos orgánicos como aglutinantes, ceras y resinas, ha requerido un análisis previo mediante espectroscopia de infrarrojo por transformada de Fourier (FIIR), para determinar los grupos funcionales de los compuestos orgánicos presentes la mezcla. Esta técnica instrumental permite elegir con posterioridad el tipo de preparación de muestras y la técnica cromatográfica a utilizar en cada caso concreto, ya que cada tipo de filmógeno (aceites, resinas o proteínas) puede requerir un camino analítico diferente. Los análisis de aglutinantes, ceras y barnices se han realizado en el Centro de Instrumentación Científica de Granada y en los laboratorios Larco Química y Arte, S.L.

La identificación de las fibras textiles del lienzo se ha llevado a cabo mediante microscopía óptica. Este estudio está basado en la observación de las características de las fibras atendiendo a su morfología, diámetro, agrupaciones, etc. y en el estudio del comportamiento de las fibras frente a diferentes reactivos químicos.

\section{RESULTADOS}

\section{Soporte}

El tejido constituyente del soporte pictórico, tanto del lienzo original como el del reentelado, es de lino.

\section{Preparación}

La pintura presenta dos capas de preparación. La primera, de color blanco grisáceo y espesor variable, está compuesta por carbonato cálcico y partículas de negro de carbón animal (y trazas

\section{Se han utilizado técnicas no destructivas para el análisis de zonas delicadas del cuadro como el rostro o las vestimentas donde no ha sido posible la toma de muestras}

de yeso y de tierras). El aglutinante es de naturaleza proteica, cola animal. Esta capa tiene la función de preparar el lienzo, nivelando las irregularidades del soporte e impidiendo que la tela absorba las capas de imprimación cuando éstas son al aceite. La segunda capa preparatoria o imprimación es de color rojo y está constituida por tierra roja (tierras ricas en óxidos de hierro, responsables del color rojo) acompañada por pequeñas cantidades de negro de carbón vegetal y aglutinada con aceite de linaza. El análisis mediante SEM-EDX de las secciones transversales sugiere la presencia de algunas partículas de cuarzo, pirita, carbonato cálcico, óxidos de hierro y óxido de titanio. En algunas muestras se ha detectado cantidades mínimas de un compuesto de plomo, empleado probablemente por su poder secativo.

En todos los puntos analizados por microfluorescencia de rayos $X_{1}$ independientemente del color de la capa pictórica final, se detecta de forma generalizada el hierro y calcio. Mediante difracción de rayos $X$ se han identificado calcita $\left(\mathrm{CaCO}_{3}\right)$ y hematite $\left(\mathrm{Fe}_{2} \mathrm{O}_{3}\right)$ como compuestos minerales comunes en todos los puntos analizados, especialmente en las zonas donde no había una presencia masiva de blanco de plomo. Esto, unido a la información extraída del estudio estratigráfico, indica que dichos elementos químicos están contenidos en las capas preparatorias de la pintura en forma de calcita y hematite.

\section{Capas pictóricas}

Dado el estado de la pintura, sin daños visibles o lagunas en donde fuera posible la extracción de muestras sin ocasionar un perjuicio a la obra, el estudio estratigráfico únicamente se ha podido realizar en los bordes de la pintura, una vez desmontado el marco. Es por ello que únicamente se han extraído micromuestras de diferentes zonas del fondo y del negro de la indumentaria del personaje. En este último caso todas las muestras extraídas han presentado similar secuencia estratigráfica. No ha sucedido lo mismo con el fondo, en el que se han apreciado algunas diferencias en la secuencia estratigráfica en función del punto de extracción de la muestra. 


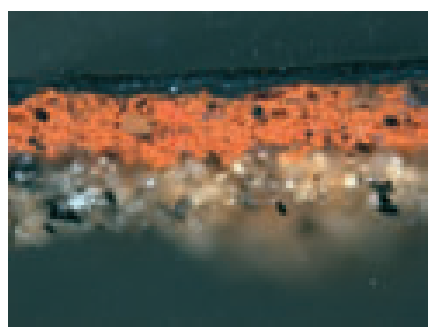

4. Sección transversal del traje negro. Foto: Lourdes Martín, IAPH

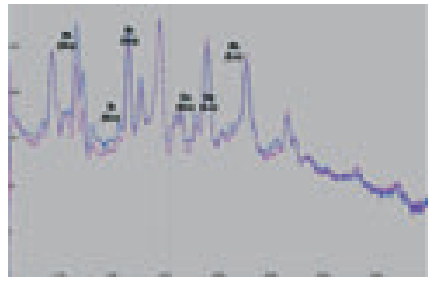

6. Espectros de FRX de la carnación frente (color violeta) y carnación frente repinte (color azul)

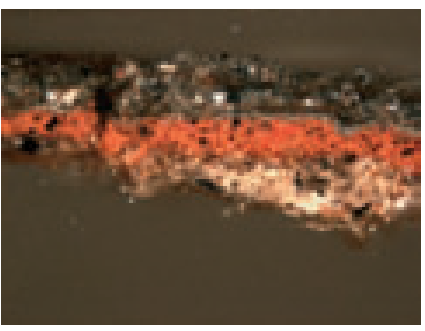

5. Sección transversal del fondo. Foto: Lourdes Martín, IAPH

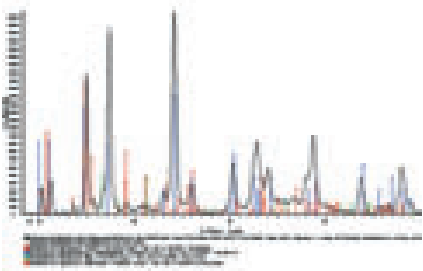

7. Diagrama de DRX recogido en la carnación frente

El estudio de las muestras de pintura del fondo (imagen 5) es más complejo ya que no todas las muestras presentan la misma secuencia estratigráfica. Las diferencias observadas se dan siempre en las capas externas.

Todas las muestras analizadas presentan, superpuesta a la imprimación roja, una fina capa de color gris claro compuesta por blanco de plomo, calcita, negro de carbón y trazas de tierras (y de azurita en algunos casos) y, generalmente, sobre la capa anterior, un estrato de color grisáceo, compuesto por blanco de plomo, calcita, negro de carbón y trazas de tierras y de sombra.

A partir de este estrato existen variaciones en la secuencia estratigráfica. Mientras que en algunas muestras se observa, superpuesta sobre las capas anteriores, una gruesa capa de carbonato cálcico (con trazas de carbón y tierras) aglutinada con cola animal, en otras muestras dicha capa no aparece. A continuación se observa, en casi todas las muestras, la presencia de dos capas pictóricas pardas. La inferior es un estrato de color pardo grisáceo compuesto por blanco de plomo, calcita, ocre, negro de carbón y trazas de sombra; la superior, de color pardo oscuro, está constituida por pardo orgánico, blanco de plomo, calcita, ocre y negro de carbón. Por último mencionar que en una de las muestras analizadas, se ha encontrado también, además de todos los estratos anteriores, un repinte oscuro de blanco de cinc mezclado con tierras y negro de carbón.

Los resultados obtenidos mediante fluorescencia de rayos $X$ de las zonas del fondo indican que los elementos químicos mayoritarios son el hierro, el calcio, el plomo y el manganeso. Por difracción de rayos $\mathrm{X}$ se identifica blanco de plomo (cerusita, $\mathrm{PbCO}_{3}$ e hidrocerusita, $\mathrm{Pb}_{3}\left(\mathrm{CO}_{3}\right)_{2}(\mathrm{OH})_{2}$ y calcita $\left(\mathrm{CaCO}_{3}\right)$; otras fases como cuarzo $\left(\mathrm{SiO}_{2}\right)$, yeso $\left(\mathrm{CaSO}_{4} \cdot 2 \mathrm{H}_{2} \mathrm{O}\right)$ e incluso carbón (C) no pueden descartarse. En las zonas del fondo rojizas, donde hay un gran desgaste de la pintura, Ios análisis por FRX detectan una masiva presencia de hierro $y_{\text {, }}$ por DRX, la presencia de hematite $\left(\mathrm{Fe}_{2} \mathrm{O}_{3}\right)$ es posible.

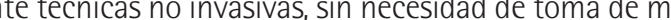
De este modo se ha podido identificar la composición química elemental y mineralógica de las carnaciones y del blanco del ropaje de Góngora, así como zonas en las que se sospechaba de la existencia de posibles repintes. No obstante, también se han aplicado a otras zonas de la pintura donde se han realizado estratigrafías para poder extraer el máximo posible de información analítica.

Para la realización de la vestimenta negra del personaje, el autor ha superpuesto sobre la imprimación roja, una capa de color negro compuesta por blanco de plomo, calcita, negro de carbón y tierras (imagen 4).

Los análisis por fluorescencia de rayos X (FRX) y difracción de rayos $x(D R X)$ realizados en dos zonas del traje negro son muy similares. Se detecta como elementos químicos mayoritarios el calcio, hierro y plomo. Por DRX se identifica blanco de plomo (cerusita, $\mathrm{PbCO}_{3}$ e hidrocerusita, $\mathrm{Pb}_{3}\left(\mathrm{CO}_{3}\right)_{2}(\mathrm{OH})_{2^{\prime}}$ calcita $\left(\mathrm{CaCO}_{3}\right)$, cuarzo $\left(\mathrm{SiO}_{2}\right)$ y pequeñas cantidades de yeso $\left(\mathrm{CaSO}_{4} \cdot 2 \mathrm{H}_{2} \mathrm{O}\right)$ en uno de los puntos analizados. No se ha detectado ningún pigmento específico para el color negro.

El halo blanquecino que rodea al personaje se ha analizado por FRX. Los análisis quimicos indican presencia de plomo, en cantidades más elevadas a las detectadas en otras zonas del fondo, y manganeso.

Mediante técnicas no invasivas se ha podido analizar zonas de la carnación que de otro modo hubiese sido imposible ante la imposibilidad de toma de muestras.

Los espectros de fluorescencia de rayos $\mathrm{X}$ en la carnación han identificado la presencia de plomo, calcio, hierro y mercurio (imagen 6). Los análisis por DRX realizados en las carnaciones han detectado la presencia de blanco de plomo (cerusita, $\mathrm{PbCO}_{3}$ e hidrocerusita, $\mathrm{Pb}_{3}\left(\mathrm{CO}_{3}\right)_{2}(\mathrm{OH})_{2}$ ), bermellón (cinabrio, $\mathrm{HgS}$ ) y pequeñas cantidades de yeso $\left(\mathrm{CaSO}_{4} \cdot 2 \mathrm{H}_{2} \mathrm{O}\right.$ ) (imagen 7 ). En la zona de las carnaciones con repintes se han identificado mediante fluorescencia de rayos $\mathrm{X}$ que además de los elementos químicos plomo, calcio, hierro y mercurio, contienen zinc y titanio (imagen 6 ). 
En la zona del bigote el análisis por fluorescencia de rayos $x$ detecta cantidades elevadas de hierro y algo de mercurio. Por difracción de rayos $x$ se identifica blanco de plomo (cerusita, $\mathrm{PbCO}_{3}$ e hidrocerusita, $\left.\mathrm{Pb}_{3}\left(\mathrm{CO}_{3}\right)_{2}(\mathrm{OH})_{2}\right)$ y posiblemente hematite $\left(\mathrm{Fe}_{2} \mathrm{O}_{3}\right)$, calcita $\left(\mathrm{CaCO}_{3}\right)$ y cuarzo $\left(\mathrm{SiO}_{2}\right)$.

Otra zona que sólo ha sido posible analizar mediante técnicas no invasivas es el blanco del cuello. Los análisis por fluorescencia de rayos $X$ detectan gran cantidad de plomo, probablemente debido a la presencia de blanco de plomo.

\section{Aglutinantes}

El tipo de aglutinante empleado en la preparación blanco-grisácea de carbonato cálcico es de naturaleza proteica, una cola de origen animal. La imprimación rojiza está aglutinada con aceite de linaza.

En las capas pictóricas se ha utilizado un aglutinante de tipo oleoso. El análisis por cromatografía de gases de algunas muestras de capas pictóricas determinó la presencia de aceites secantes. Las proporciones entre los ésteres metílicos de los ácidos palmítico y esteárico indican que se trata de aceite de linaza.

Las diversas intervenciones posteriores analizadas han sido realizadas también empleando como aglutinante aceite de linaza.

La espectroscopia infrarroja por transformada de Fourier reveló en la muestra correspondiente a una zona expuesta de la superficie de la obra (es decir, no cubierta por el marco) un espectro en el que se puede apreciar la presencia de cola animal procedente posiblemente de una antigua consolidación.

\section{Barnices}

Se tomaron varias micromuestras de la superficie con el fin de conocer la naturaleza de los recubrimientos orgánicos de la superficie pictórica.

A partir de los raspados superficiales tomados en diferentes puntos de la obra se determinó la composición del barniz actual. El análisis por GC/MS de la muestra en polvo obtenida reveló un contenido en ácidos grasos (ac. palmítico, ac. esteárico, ac. subérico y ac. acelaico) y terpenos (ac. dehidroabiético y ac.7-oxodehidroabiético). Estos compuestos ponen de manifiesto la existencia de una resina de conífera, probablemente colofonia y trazas de aceite de linaza (imagen 8).

Se extrajo asimismo un raspado superficial en uno de los laterales del cuadro, de un repinte constituido por un barniz pigmentado para determinar su naturaleza. El estudio mediante CG/MS reveló un contenido en ácidos grasos (ac. palmítico, ac. esteárico y ac. acelaico) y terpenos -ácido dehidroabiético (DHA) y ácido 7-oxodehidroabiético (7-oxo-DHA)-, compuestos que indican la presencia de una resina de colofonia con trazas de aceite secante.

La eliminación de las capas de suciedad y barniz de esta obra presentaba ciertas dificultades al estar recubriendo el barniz una capa pictórica muy fina (hay zonas en las que se transparenta la preparación rojiza), motivo por el que la restauradora requirió al equipo de laboratorio la realización de un test de solubilidad previo a las prueba de limpieza, con el fin de garantizar que la mezcla de disolventes sólo provoque la remoción del barniz, sin alterar ni dañar la capa de pigmentos aglutinados al aceite. Por ello, una vez determinada la composición del barniz empleado en la pintura, se aplicó el test de solubilidad basado en el Triángulo de Teas para tratar de encontrar los sistemas de disolventes más adecuados para su eliminación.

\section{ESTUDIO MEDIANTE TÉCNICAS DE EXAMEN POR IMAGEN, ESTADO DE CONSERVACIÓN Y TRATAMIENTO}

En el estado de conservación de la obra ha influido no sólo la degradación natural de los materiales que la componen, sino las condiciones ambientales a las que ha estado sometida, así como las intervenciones anteriores realizadas para su conservación.

La obra presenta una antigua restauración, y según su historia material enviada por La Fundación Lázaro Galdiano, estos tratamientos son anteriores a la formación del Museo en 1951. Pueden estar ejecutados por uno de los dos pintores-restauradores que trabajaron desde 1894, Juan Alaminos López o su hijo José Alami-

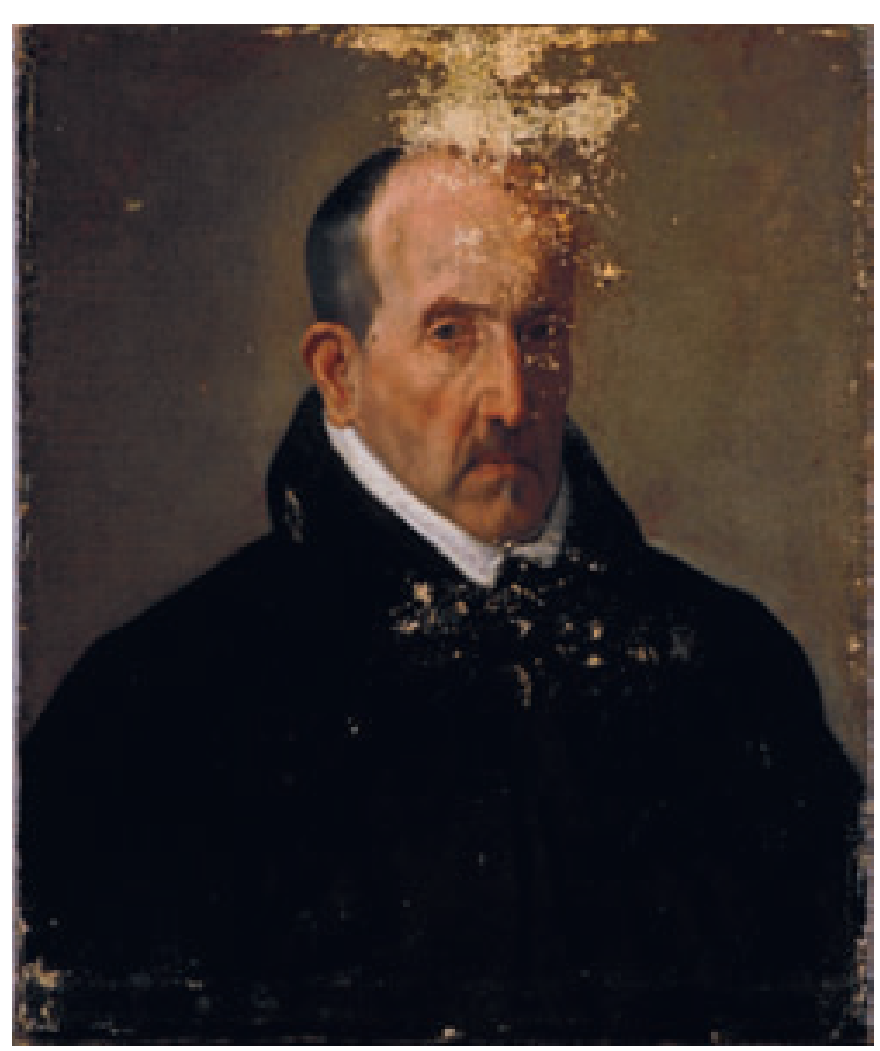

9. Fase de estucado. Foto: Eugenio Fernández Ruiz, IAPH 

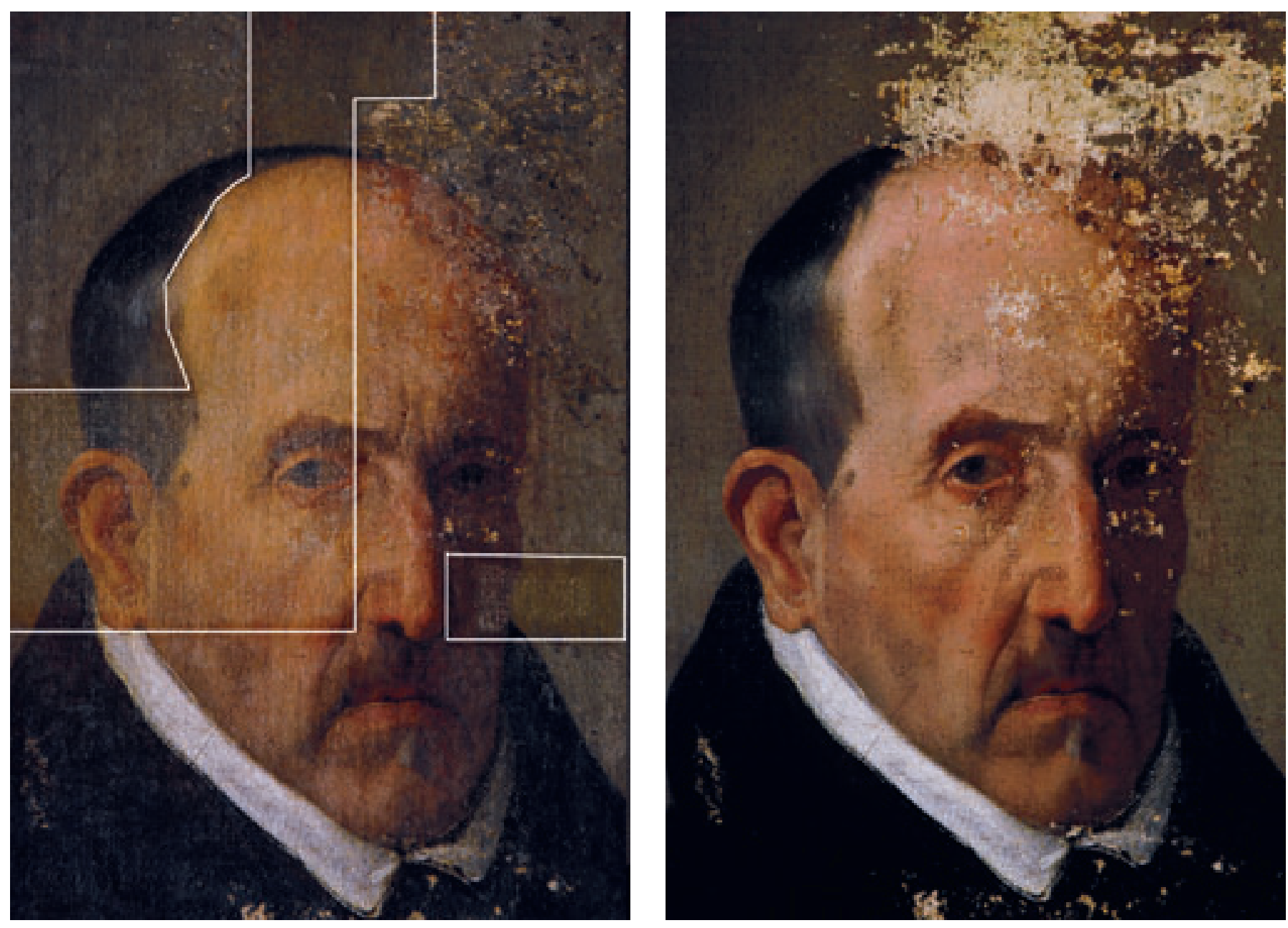

10. Fases del proceso de intervención: limpieza con testigos de suciedad, estucado, reintegración cromática y estado final. Fotos: Eugenio Fernández Ruiz, IAPH

nos Alamitos, que se formó junto a su padre y Enrique Martínez Cubells, forrador del Museo del Prado, que realizaron las siguientes operaciones: reentelado a la gacha con la colocación probablemente de un bastidor nuevo, eliminación del barniz envejecido, estucado, reintegración y barnizado.

\section{Bastidor}

No es el original, pertenece a la última intervención en la que se realizó un reentelado de la obra, ya que por sus características (tener el borde interior rebajado) no corresponde a los realizados en el s. XVII. Además, los bordes de la tela original situados en los cantos del bastidor poseen película pictórica.

El material utilizado es madera de pino y el tipo de corte es Iongitudinal. Se trata de un bastidor con cuñas, de forma y sección rectangular con un travesaño horizontal formando dos cuadrantes. El tipo de ensamble de los ángulos entre largueros es machihembrado, mientras que entre travesaño y larguero es en forma de horquilla, ambos con caja para la cuña. Las medidas del larguero horizontal es de $45 \mathrm{~cm}$ y el larguero vertical es $54 \mathrm{~cm}$. Ambos con un ancho de $3,5 \mathrm{~cm}$ y un grosor de $1,5 \mathrm{~cm}$. El travesaño tiene $38,5 \mathrm{~cm}$ de largo, 3,3 cm de ancho y $1,5 \mathrm{~cm}$ de grosor. El sistema de montaje del bastidor en el marco es a través de cuatro flejes metálicos que parten desde el marco al bastidor.
El travesaño posee una grieta que va desde la caja para la cuña hacia su parte central y tiene una longitud de $5 \mathrm{~cm}$ aproximadamente. Debido a esta fisura el travesaño está desplazado entre 2 ó $3 \mathrm{~mm}$ hacia el exterior en el lado izquierdo. El estudio radiográfico nos aporta además de todos los datos anteriormente señalados el sentido de la veta de la madera y la profundidad de las tachuelas de montaje del soporte al bastidor.

El bastidor se encuentra en magnífico estado de conservación ya que cumple las funciones de sustento y tensado de la obra por lo que se ha mantenido el mismo y se ha realizado sólo un tratamiento de conservación consistente en una limpieza mecánica-química de depósitos superficiales y encolado de la fisura del travesaño.

\section{Soporte pictórico}

La obra se encuentra reentelada. La tela original dobla el borde del bastidor teniendo película pictórica sobre ella en esta zona, luego se puede ver que el bastidor original era de mayor tamaño que el actual. El tejido de refuerzo es de un tono blanco amarillento, muy regular y sin nudos. La fibra es de lino en ambos casos.

El número de hilos de trama y urdimbre en la tela original es de 9 por 11. La poca densidad de la tela coincide con las telas utilizadas 


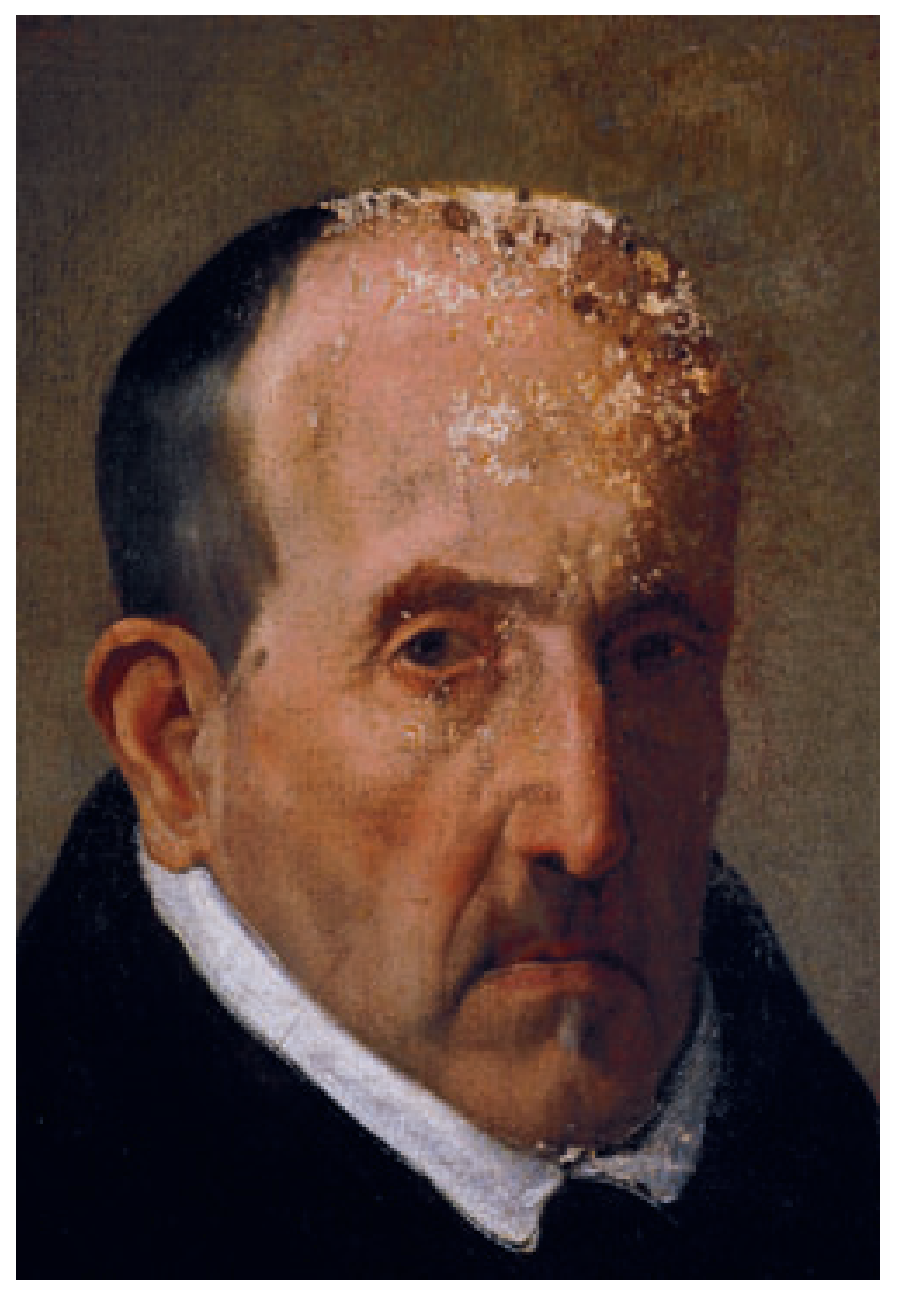

cronológicamente en fecha anterior a Los borrachos del Museo del Prado (1628) (GARRIDO PÉREZ, 1992: 57).

El número de hilos del tejido de refuerzo es de 18 de trama por 19 de urdimbre por $\mathrm{cm}^{2}$. El tipo de armadura de ambas es tafetán, un ligamento simple cuyo curso se limita a dos hilos de urdimbre y a dos pasadas de trama, según el cual los hilos pares y los impares alternan a cada pasada por debajo y por encima de la trama. Esto se ve por el reverso en la tela de reentelado y en el anverso a través de la película pictórica.

El soporte original está constituido por una sola pieza de $47 \mathrm{~cm}$ de ancho por $56 \mathrm{~cm}$ de largo aproximadamente. La tela de reentelado tiene $51 \mathrm{~cm}$ de ancho por $60 \mathrm{~cm}$ de largo. La obra se encuentra bien tensada con un sistema de montaje al bastidor que consiste en tachuelas dispuestas por el borde y adhesión con gacha por el reverso con las esquinas recortadas.

El examen con luz tangencial ratifica que no hay deformaciones del soporte ni separación entre los tejidos. El estudio radiográfico confirma que el tipo de armadura de la tela original es tafetán, aunque existe una zona en la que aparece una deformación del ligamento, es decir, cambia la densidad de su construcción interna. Esto puede ser motivado tanto por la irregularidad de los hilos, como a la hora de su ejecución manual en el telar. También

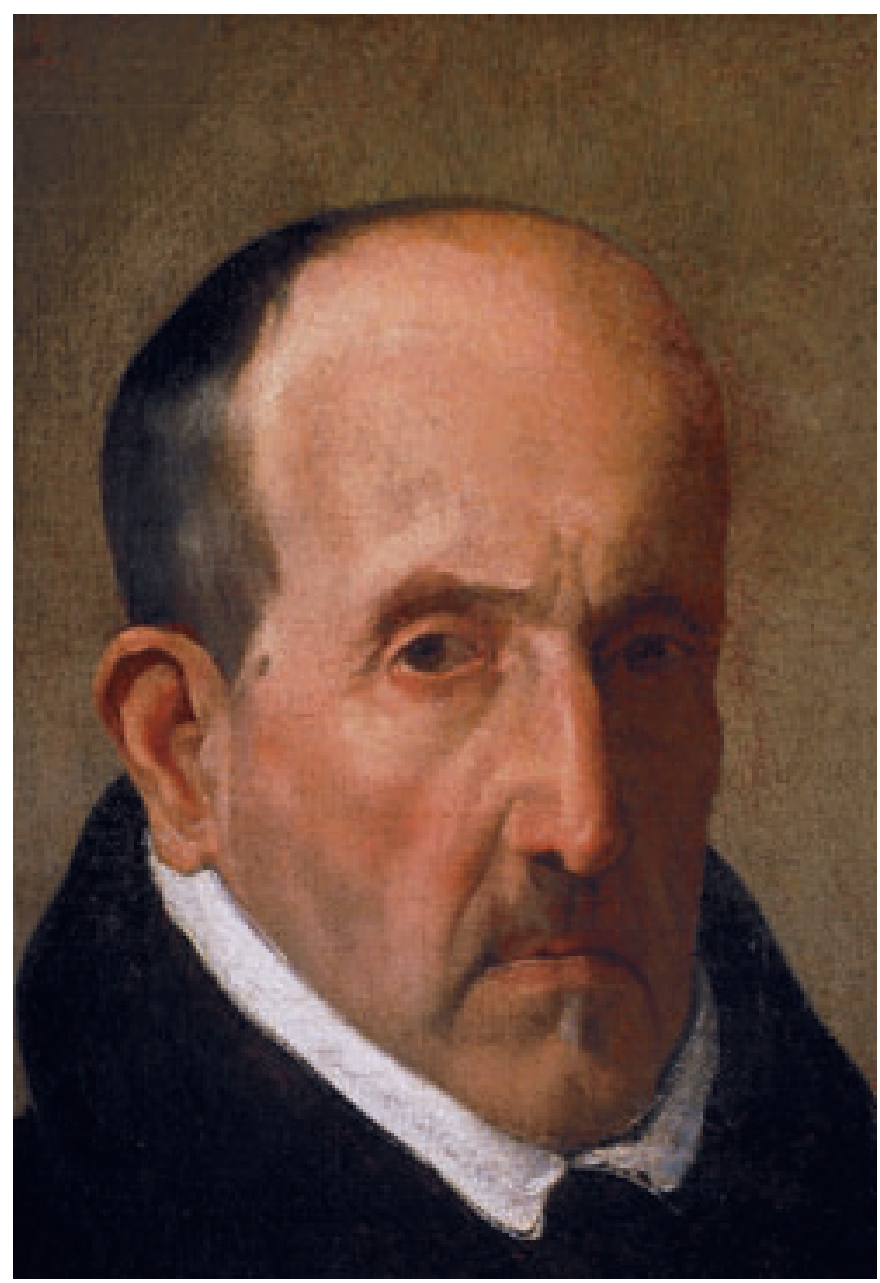

se puede observar que está constituida por una sola pieza y que el sistema de montaje en el bastidor se realiza mediante tachuelas equidistantes entre sí. La tela de refuerzo ha fortalecido la original, pues presentaba fragilidad y una rotura en la zona del cuello, por lo tanto ambas se encuentran actualmente en buenas condiciones de conservación y únicamente se ha realizado una limpieza mecánica del reverso para la eliminación de depósitos superficiales respetando la inscripción $F 11995$ en el reverso de la tela de refuerzo escrita con tiza ya que no daña la obra.

\section{Capa de preparación e imprimación}

La aplicación de esta capa fue realizada mediante brocha. La preparación es blanca y sobre ella se encuentra una imprimación rojiza. Las lagunas se pueden localizar en la parte superior y central del lienzo, y ver su extensión exacta con facilidad en la radiografia, completando el examen visual realizado en la obra.

Al coincidir las lagunas de la película pictórica con las de la preparación, se puede suponer que ambas forman una unidad, por lo que las tensiones del soporte pictórico afectan directamente a estos estratos superiores creando cuarteados y levantamientos. Todo esto, junto con la humedad, es probablemente el motivo principal, en el pasado, del daño ocurrido en la zona superior y central. Al encontrarse mojada y a su vez enrollada, puesto 


\section{El dato más interesante \\ que desvela el estudio \\ radiográfico del retrato es un \\ arrepentimiento en la zona del \\ cuello de la indumentaria}

que los daños se presentan equidistantes y de mayor a menor tamaño, además de estar las capas unidas entre sí, se produjo un desprendimiento de ambas, apareciendo asi las lagunas que se veían estucadas y reintegradas por intervenciones anteriores (según la historia material fue anterior a la formación del Museo en 1951).

No existe documentación de fijaciones puntuales realizadas posteriormente al daño anterior, por lo tanto era muy probable que el motivo del desprendimiento durante el traslado para la exposición de Granada fuese una falta de adhesión con el soporte, agravado por un cambio de temperatura-humedad, sin descartar algún pequeño golpe. En la exposición se realizó un tratamiento de urgencia, que consistió en la fijación con acetato de polivinilo del pequeño fragmento de la capa de preparación y película pictórica al soporte.

En la parte superior de la obra, en el fondo sobre el rostro, se observó un levantamiento en una grieta con peligro de desprendimiento. Ésta era una zona bastante delicada con abundantes estucos de intervenciones anteriores que corresponde con el primer daño de la preparación expuesto anteriormente. El estudio realizado con luz rasante confirmó el levantamiento de esta zona. En el borde superior y en el lateral derecho pudo apreciarse una pequeña línea de estuco blanco producida por el roce del marco.

El tratamiento realizado ha consistido en la fijación de la zona superior con esmero y como prevención de todo el lienzo. Para ello se ha utilizado el método tradicional con coleta y papel de seda, aplicando presión y calor en la zona a fijar.

Durante la restauración se han encontrado dos tipos diferentes de estuco, realizados en distintas intervenciones, uno de color gris y otro blanquecino. De ambos se han eliminado sólo los que invaden la pintura original, respetando así la mayoría de ellos y aplicando pequeños estucos nuevos, compuestos de sulfato de cal y cola de conejo.

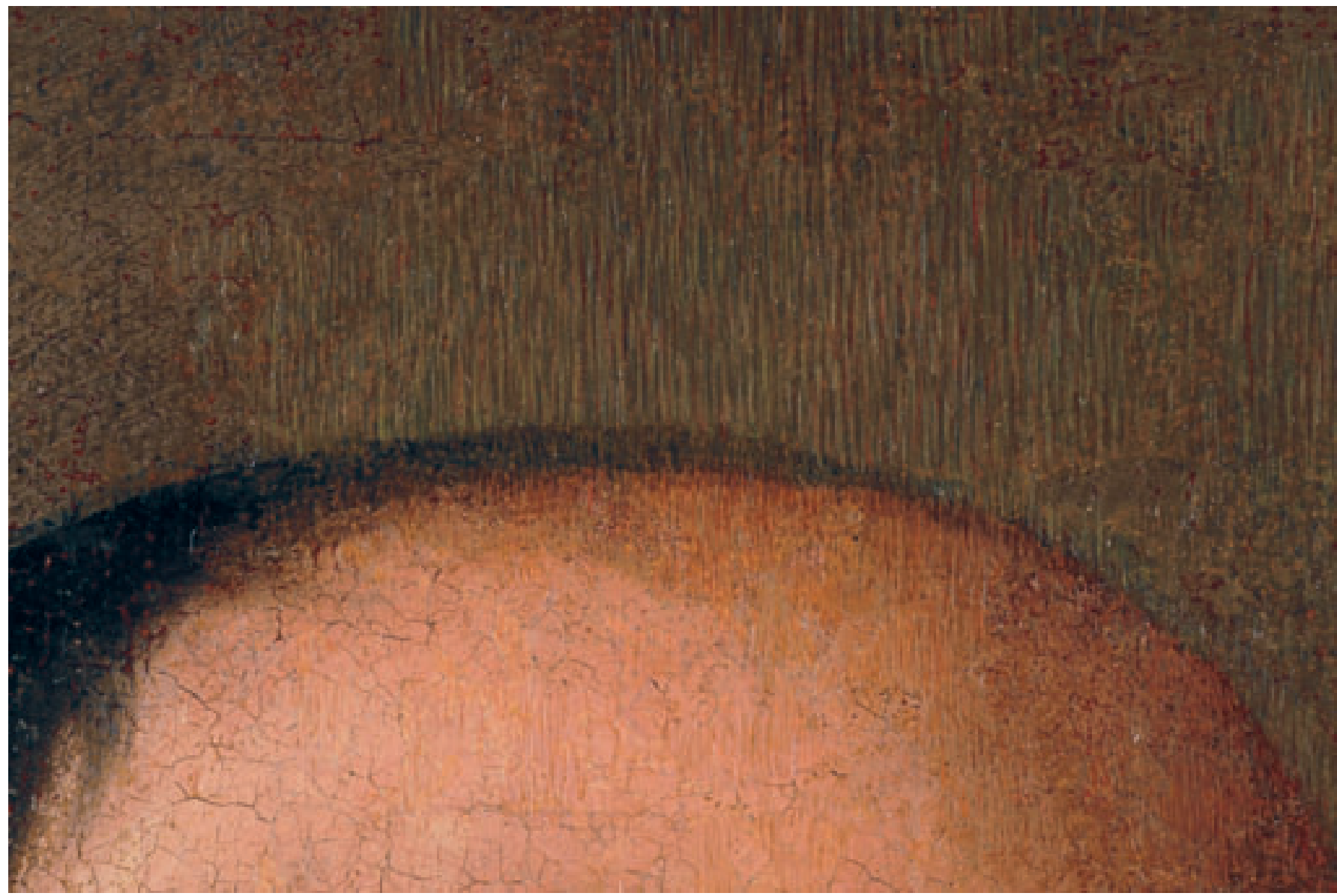

11. La reintegración cromática de lagunas de la película pictórica se ha realizado mediante la técnica del rigattino. 


\section{Capa de película pictórica y capa de protección}

El material constitutivo de la película pictórica es el óleo, por el brillo, textura y transparencia que presenta el pigmento aglutinado con aceite. Nos encontramos ante una pintura de ejecución estudiada, segura y rápida, con carga en el pincel en las zonas de luz y con poca en las zonas de los ropajes y el fondo. La paleta de color es básica aun siendo un retrato, utilizando rojos y ocres para la encarnadura; blancos y negros para la indumentaria y el fondo, logrando representar así al personaje con toda su humanidad, tanto en grandeza como en debilidad, obteniendo al mismo tiempo una luz difusa que envuelve suavemente al retratado.

En un primer examen visual se distingue un cuarteado menudo, provocado por la transmisión del movimiento del soporte a la capa de preparación y de ésta a la de la película pictórica, repartido por toda la superficie y logrando una textura lisa.

El estudio radiográfico pone de manifiesto los materiales y la técnica del artista para conseguir plasmar al modelo. La manera de aplicar el color del fondo para encajar la figura es mediante una gran pincelada segura, rodeando las zonas de más luz del rostro y en las sombras, partiendo desde la figura hacia el exterior, delimitando al personaje dentro del conjunto. Esto es similar a lienzos de
Velázquez, como es el caso del Retrato de un hombre joven, entre otros (GARRIDO PÉREZ, 1992: 114-116).

El dato más interesante del retrato es un arrepentimiento en la zona del cuello de la indumentaria. En un primer planteamiento compositivo éste es estrecho y ceñido al personaje, pero al final el autor lo cambia por una solapa amplia con un discreto cuello blanco de camisola. Este dato es de suma importancia porque si la obra que se está estudiando es una copia, ¿qué quiere decir ese arrepentimiento?

El movimiento de las pinceladas puede seguirse comenzando en la zona donde posee más carga el pincel y en dirección a la de menos, dando un reflejo pormenorizado de la manera de ejecutar la obra. Asi se ve cómo en el traje las pinceladas van hacia abajo sueltas y rápidas para crear los pliegues. En el fondo se ve una pincelada detrás del hombro izquierdo hacia abajo con la finalidad de crear atmósfera por detrás del personaje, delimitarlo y centrarlo en el lienzo.

El rostro tiene mucha fuerza y contraste al utilizar blanco de plomo en su ejecución. Las pinceladas son directas, creando planos como en el entrecejo, nariz y pómulo, y aprovecha la transparencia en la pincelada para que salga la imprimación en las zonas de sombra.
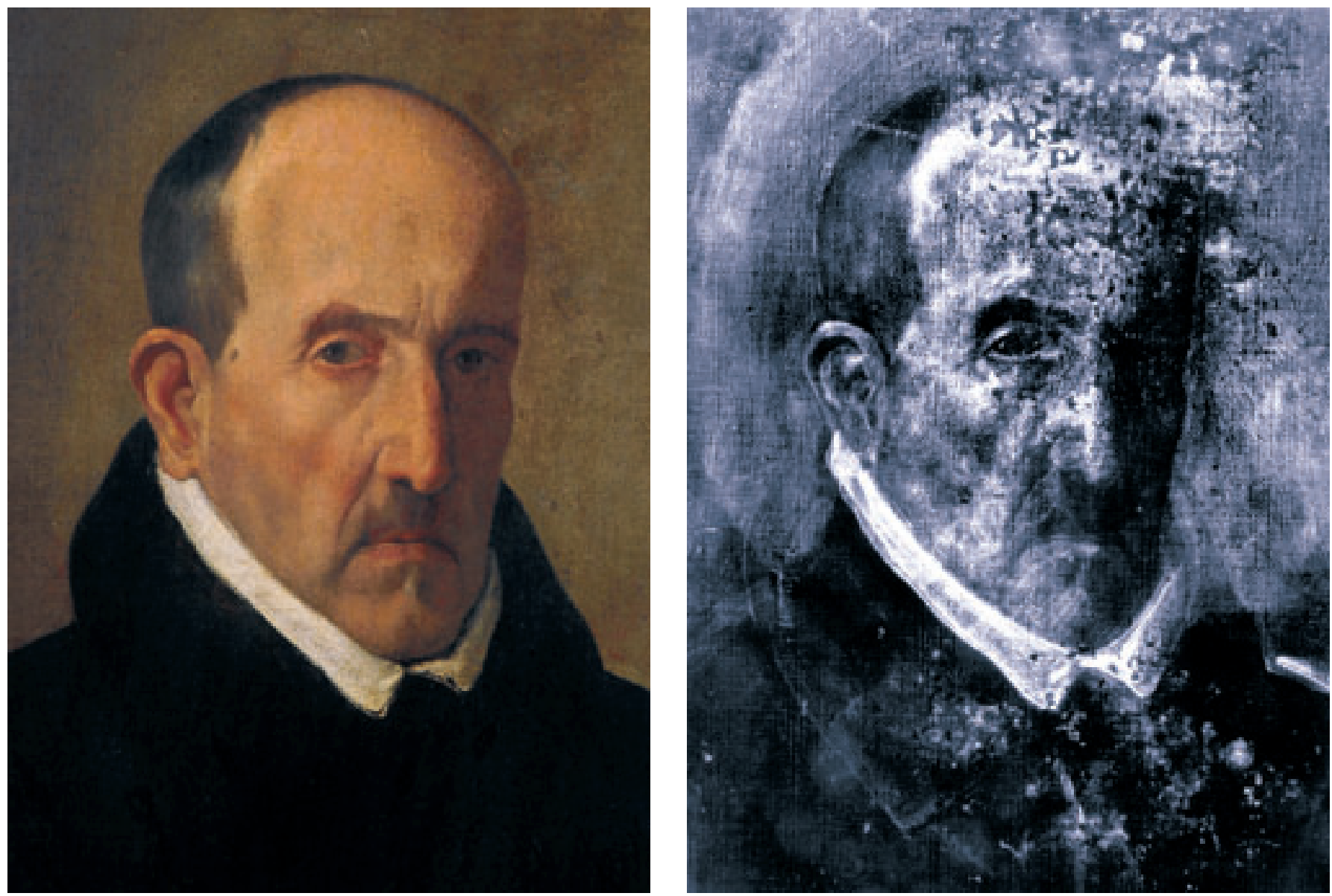

12. Estado inicial y radiografía en la que se observa el arrepentimiento del cuello de la vestimenta. Foto: Eugenio Fernández Ruiz, IAPH 

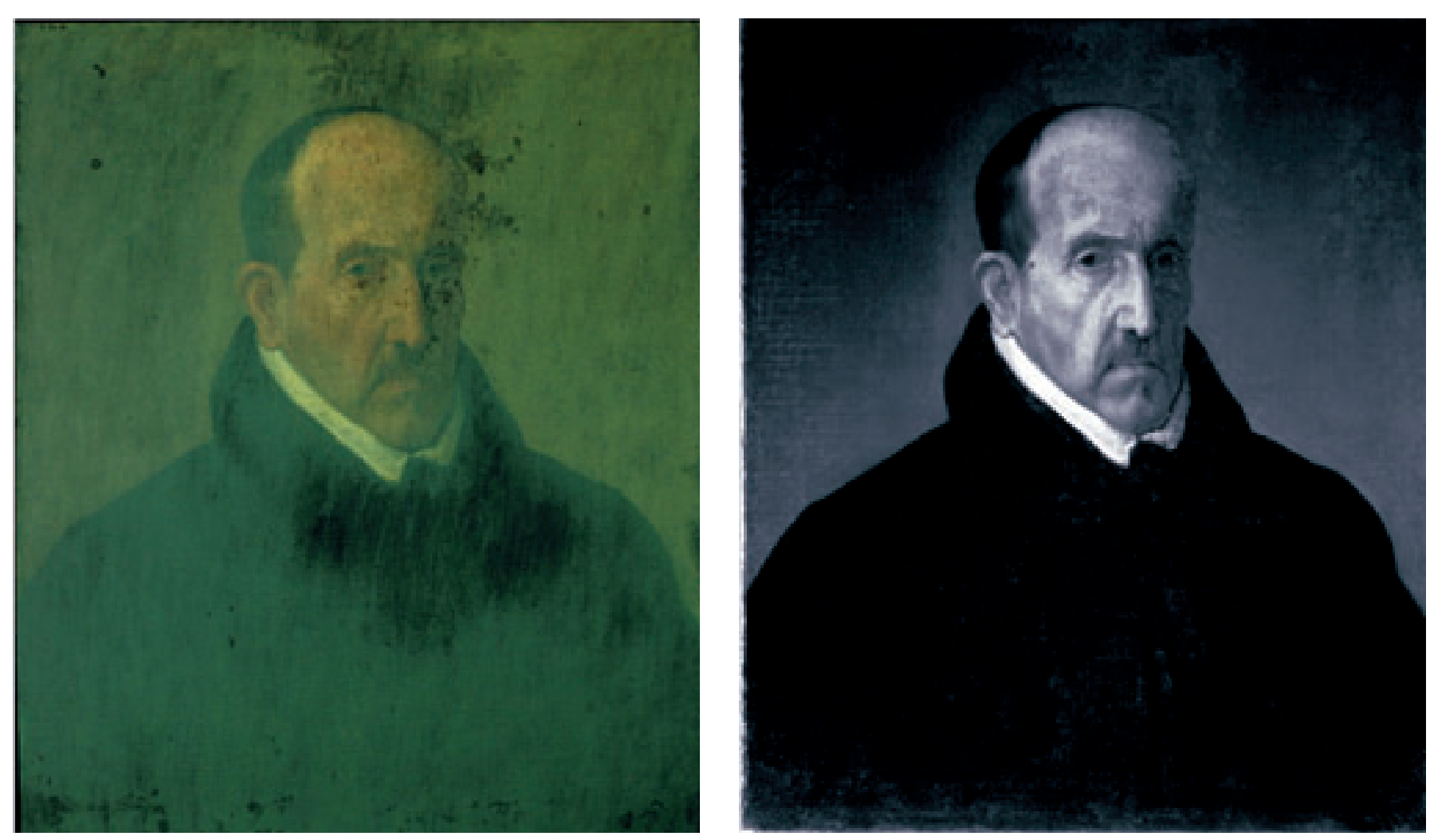

13. Fotografía con luz ultravioleta e imagen infrarroja. Foto: Eugenio Fernández Ruiz, IAPH

El estudio realizado con luz infrarroja corrobora la dirección de las pinceladas de la vestidura. Comparando la radiografía con la obra se puede apreciar que el cuello de la camisola es más grande en la primera de lo que se ve realmente. En primer lugar realiza el cuello blanco y lo ajusta invadiéndolo con el negro del traje.

Tras un examen pormenorizado mediante luz ultravioleta se observaron repintes, de los que se pudo conocer su extensión exacta y localización. La distinta absorción de la luz ultravioleta en los repintes, separados por capas de barniz, hizo posible diferenciar los que fueron aplicados en último lugar, correspondiendo éstos a las últimas intervenciones sobre la obra, como en la mitad del rostro izquierdo que está en sombra, pequeñas reintegraciones en ojo, pómulo, nariz, bigote, en la zona inferior del lienzo y en el fondo.

Bajo el cuello se observó una gran zona de repintes. Se encontraron reintegraciones anteriores a éstas en la zona de la frente, nariz del personaje y en el fondo sobre el mismo lugar donde sufrió el percance. Se puede decir que esta parte no había recibido tratamiento de conservación-restauración recientemente, por lo que era más delicada que el resto de la obra.

La alteración cromática era general en toda la superficie por la oxidación del barniz de protección, dando a la obra un aspecto de color ambarino, que impedia apreciar su cromatismo original. También se veía este tipo de alteración en repintes que habian virado de color con el tiempo.

La manera de ejecutar la obra directa y ligera, llegando a utilizar

la imprimación rojiza como base en las zonas oscuras, ha reper- cutido en que los desgastes localizados en casi toda la superficie de la obra sean en menor cantidad en los lugares con mayor empaste como son las luces del rostro y el blanco del cuello de la camisola.

El estudio realizado con luz rasante ponía de manifiesto que los levantamientos de la capa de color coincidian con los de la capa de preparación y de barniz, y se podían localizar en la parte superior del fondo, en la zona donde se había producido el percance anteriormente citado. Aparecen dos huellas dactilares en el barniz en la parte superior derecha de la cabeza.

Como conclusión se puede decir que la película pictórica se encuentra bien cohesionada con los distintos estratos llegando a formar un cuerpo único.

La limpieza de la superficie pictórica ha sido el tratamiento más delicado. Para ello, habiendo conocido los resultados de la analítica referentes al tipo de pigmento, número de estratos y capa de barniz, con ayuda de la lupa binocular, se realizaron los test de solubilidad con disolventes de menor a mayor intensidad de actuación. Se decidió utilizar para la eliminación del barniz de coIofonia con aceites secantes oxidado y de repintes la mezcla de Isooctano e Isopropanol al 50\% y en zonas puntuales Tolueno e Isopropanol al 50\%.

Se realizaron catas de limpieza, eligiendo zonas con repintes y sin ellos. La dificultad que entrañaba la limpieza ocasionada por la utilización de la preparación como parte de la película pictórica, la cantidad de desgastes e intervenciones anteriores, unidas 
al escaso grosor de la película pictórica obligaba a una limpieza meticulosa con lupa, realizando varios testigos de suciedad.

Una vez terminada la limpieza se barnizó la película pictórica y los estucos se reintegraron con pigmentos al barniz a base de líneas y puntos con la finalidad de poder apreciarlos de cerca por el ojo humano, pero que de lejos se fundan éstos en la retina ofreciendo una lectura global de la obra para evitar la distracción que pudieran ofrecer las lagunas. Con objeto de no enmascarar el original se han respetado la mayoría de los desgastes.

\section{CONCLUSIONES}

Con respecto a la historiografía se podría decir, según la crítica más especializada y actual, que tanto el retrato del Museo de Boston como el de la Fundación Lázaro Galdiano son considerados casi por unanimidad obras realizadas por el propio Velázquez, aunque sin poder asegurar qué versión fue la primera, la pintada en 1622, para confirmar al fin, cuál es el primer y original retrato de Don Luis de Góngora y cuáles son réplicas o copias.

Para la realización del estudio científico se han empleado la combinación de distintos tipos de técnicas que han permitido la identificación de la variedad cromática empleado por el autor, así como la técnica de ejecución.

La preparación empleada en la obra consta de dos capas: una preparación blanquecina, de carbonato cálcico y negro de carbón animal (y trazas de yeso y de tierras) aglutinado con cola animal, y una imprimación roja, constituida por tierra roja, con pequeñas cantidades de negro de carbón vegetal aglutinada con aceite de linaza.

Los blancos analizados están compuestos exclusivamente por blanco de plomo. Las carnaciones contienen bermellón como pigmento responsable del color. Se han identificado óxidos de hierro en la zona, pero al no disponer del estudio estratigráfico es imposible saber si este compuesto se localiza exclusivamente en la capa preparatoria rojiza o también está presente en la capa de carnación junto al bermellón.

El traje negro se ha realizado con blanco de plomo, calcita, negro de carbón y tierras.

El fondo está compuesto por una capa fina con blanco de plomo, calcita, negro de carbón y trazas de tierras a la que se superpone, en algunas muestras, otra capa de color pardo grisáceo, compuesto por blanco de plomo, calcita, negro de carbón y trazas de tierras y de sombra. Sobre estas capas se han encontrado diversos repintes, algunos con estuco intercalado y otros sin él.

En todas las capas pictóricas se ha utilizado como aglutinante el aceite de linaza.
El estudio comparativo de los materiales y técnica de ejecución empleados en el retrato de Góngora con otras obras analizadas de Velázquez, de su primera etapa madrileña (1622/23-1628), recogidas en la bibliografía (NEWMAN, 1993; GARRIDO PÉREZ, 1992), muestra grandes similitudes.

Esto es especialmente significativo en el caso de las dos preparaciones, la primera blanco grisácea y la segunda roja, típicas de esta etapa del pintor. La apariencia y composición química de ambas preparaciones es practicamente idéntica a la encontrada en otras obras analizadas de este pintor, correspondientes a dicha etapa, como Retrato de hombre joven, Felipe IV (Prado, 1182) o El Infante Carlos.

Tras el tratamiento y estudios realizados mediante técnicas de imagen, se puede concluir que las dimensiones actuales de la obra variaron en intervenciones anteriores con respecto a las originales ya que las zonas de montaje del soporte sobre el bastidor, en los cantos exteriores, poseen restos de película pictórica.

La poca densidad de la tela, 11 de urdimbre por 9 de trama, coincide con las telas utilizadas cronológicamente anterior a Los borrachos(1628) (GARRIDO PÉREZ, 1992: 57).

El estudio radiográfico ha sido crucial en la localización y cuantificación de los deterioros y ha facilitado un examen más exhaustivo de la dirección y magnitud de la pincelada, pudiendo encontrar similitudes en la manera de encajar la figura en el lienzo con las obras estudiadas radiográficamente de Velázquez, como el Retrato de un hombre joven del Museo del Prado entre otros (GARRIDO PÉREZ, 1992: 114-116). Además, ha arrojado un dato muy interesante que se encontraban oculto, el arrepentimiento en la zona del cuello de la vestimenta. El cambio en el planteamiento de parte de una obra es crucial para que se realicen nuevas preguntas sobre ella, ya que si es una copia estos problemas no se los cuestiona el pintor, pues se limita a reproducir el original, luego si existe un cambio compositivo... ¿es copia?, ¿es la primera versión?

La cantidad de desgastes, repintes y la utilización de la preparación como parte del color indicaba que cualquier tratamiento era bastante delicado. El daño producido en el traslado para la exposición unido al grueso barniz oxidado, causando una alteración cromática, ha demandado por un lado una línea conservativa para recuperar la cohesión entre los distintos estratos con la finalidad de eliminar los factores causantes de los daños y en segundo lugar un tratamiento de restauración de la obra.

Gracias a la retirada de barnices oxidados y al respeto a la hora de reintegrar lagunas y desgastes, se ha logrado la recuperación de la atmósfera del fondo y los matices de la encarnadura del rostro acentuando el retrato psicológico del personaje. 


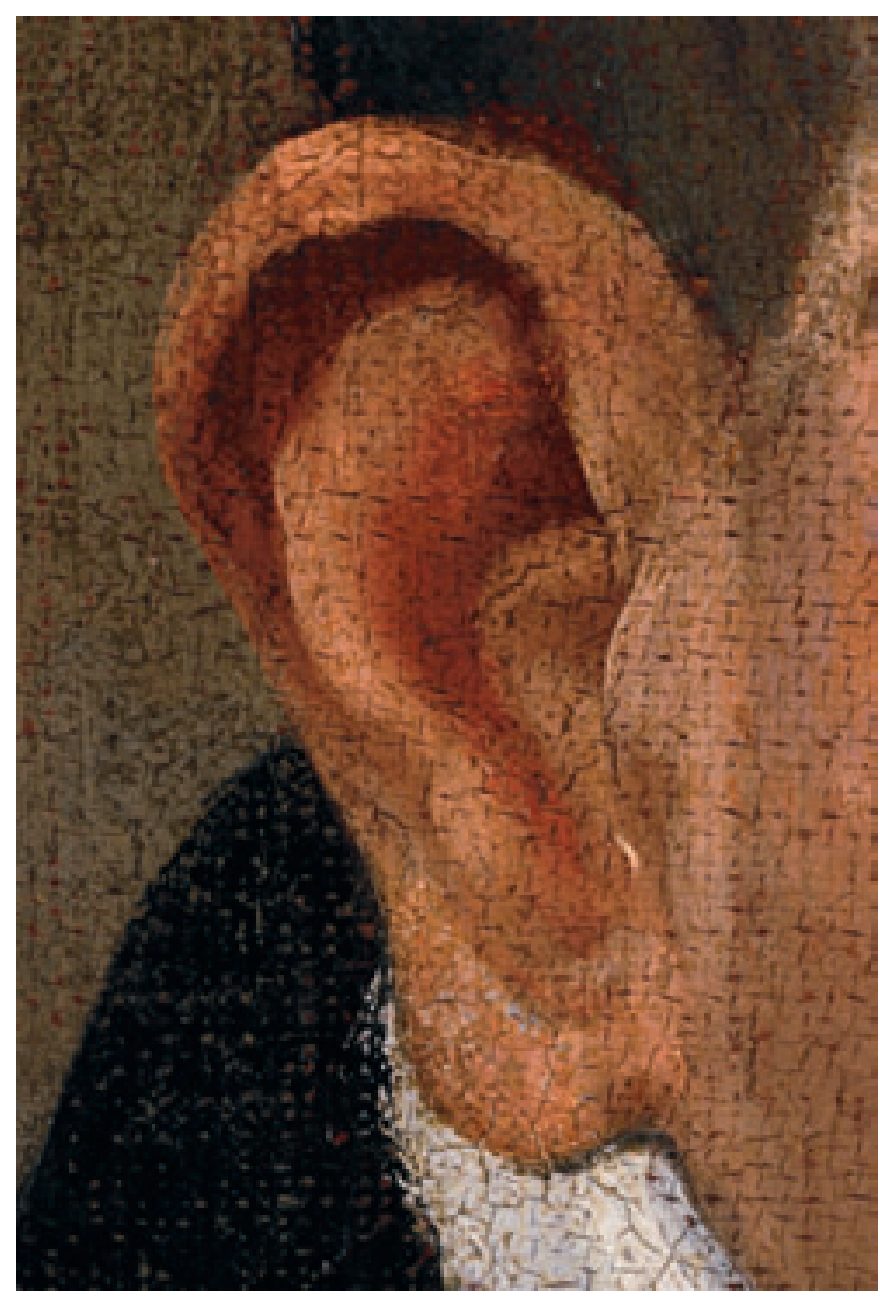

14. En esta imagen se puede apreciar la trama de la tela a través de la película pictórica. Foto: Eugenio Fernández Ruiz, IAPH

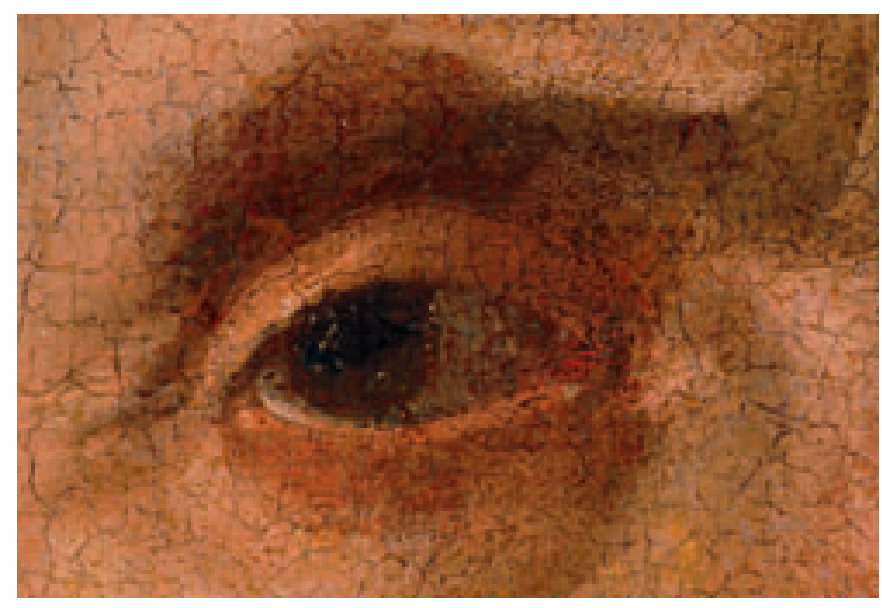

16. Detalle de las pinceladas para dar luz en párpado, iris y sobre la ceja del ojo. Foto: Eugenio Fernández Ruiz, IAPH

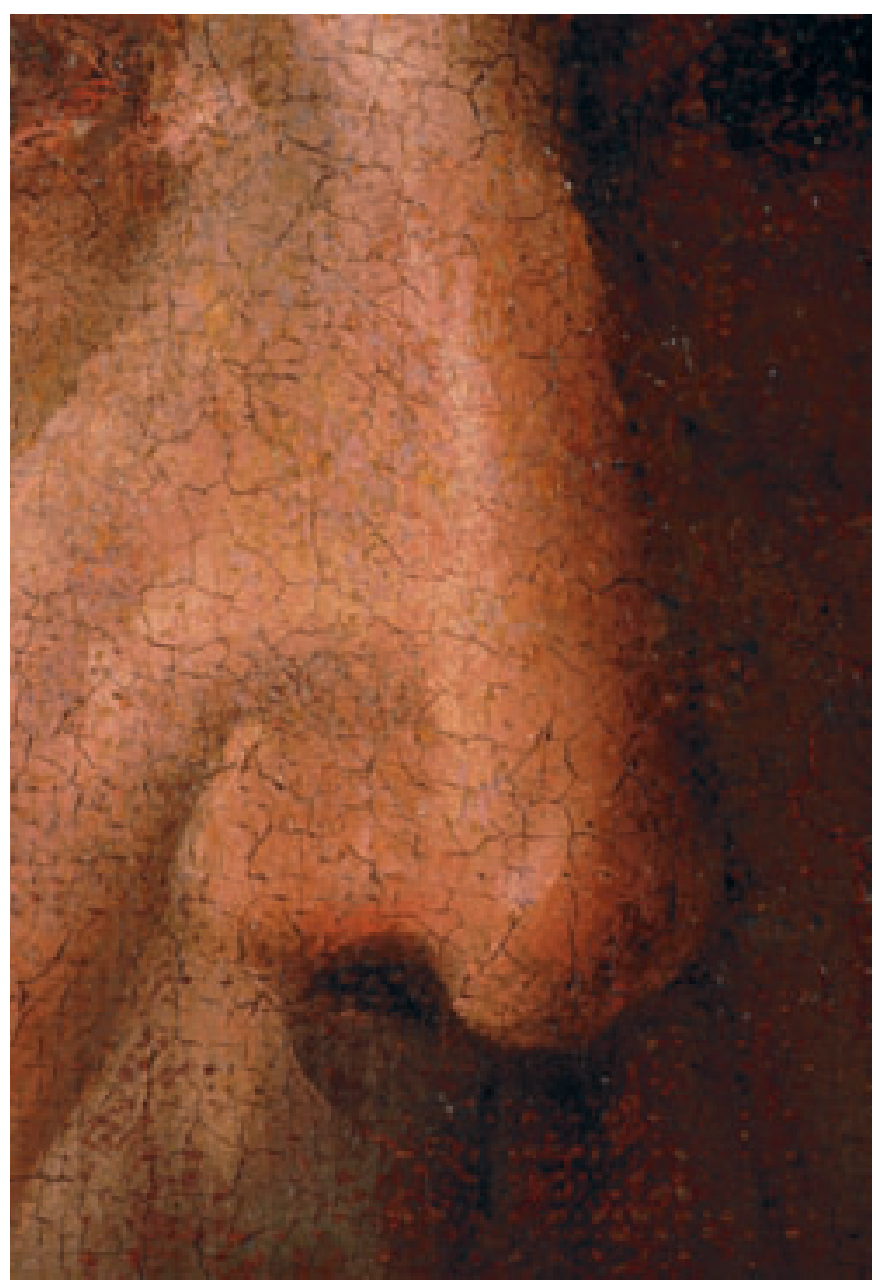

15. Detalle de la nariz tras la restauración de la obra. Foto: Eugenio Fernández Ruiz, IAPH

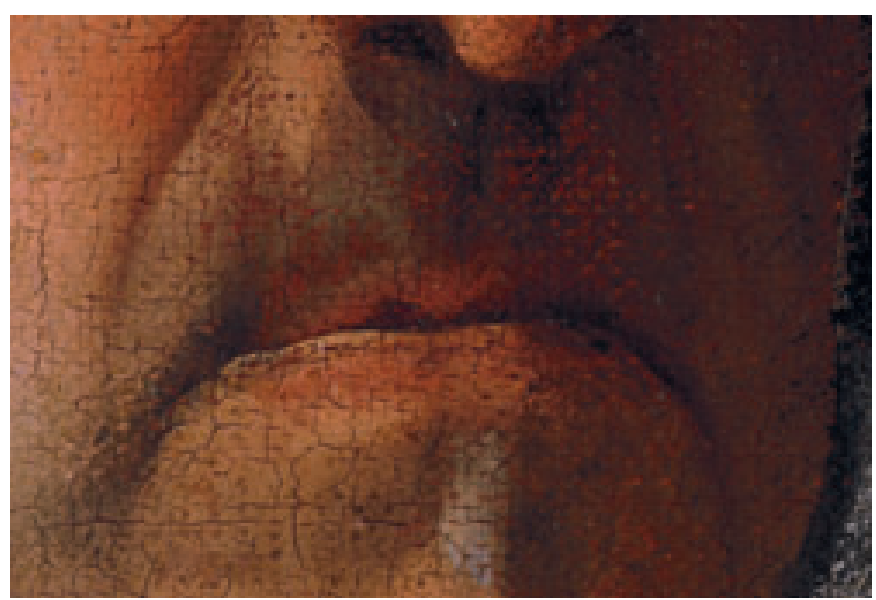

17. Detalle de la imprimación rojiza formando parte de las sombras en el bigote. Foto: Eugenio Fernández Ruiz, IAPH

Se ha logrado la recuperación de la atmósfera del fondo y los matices de la encarnadura del rostro acentuando el retrato psicológico del personaje 


\section{FICHA TÉCNICA}

Proyecto

Restauración de Retrato del poeta

D. Luis de Góngora y Argote

\section{Román Fernández-Baca Casares}

Director del IAPH

\section{Lorenzo Pérez del Campo}

Jefe del Centro de Intervención

\section{Araceli Montero Moreno}

Jefe del Área de Tratamiento

\section{María del Mar González González}

Jefe del Departamento de Talleres de Bienes Muebles

\section{Conservación-Restauración}

Lourdes Núñez Casares

Restauradora del taller de pintura del Centro de Intervención del IAPH

\section{Estudio histórico-Artístico}

Gabriel Ferreras Romero

Historiador del Arte del Centro de Intervención del IAPH

\section{Radiografía y fotografía}

Eugenio Fernández Ruiz

Jefe de Proyectos de Técnicas de Examen por Imagen del Centro de Intervención del IAPH

\section{Análisis Químicos}

Lourdes Martín García

Química del Centro de Investigación y Análisis del IAPH

Auxiliadora Gómez Morón

Química del Centro de Investigación y Análisis del IAPH

Jacques Castaing

DR Centre de Recherche et de Restauration des Musées de France CNRS

Adrián Durán Benito

Investigador postdoctoral del Centre de Recherche et de Restauration des Musées de France CNRS

Ángel Jesús Polvorinos del Río

Dpto. de Cristalografia, Mineralogía y Química Agrícola de la

Universidad de Sevilla

Larco Química y Arte, S.L

\section{Notas}

${ }^{1}$ Datos facilitados por la conservadora Amparo López de la Fundación LázaroGaldiano de Madrid.

${ }^{2}$ Datos facilitados por la viuda de Ramón Aras Jáuregui, propietaria de la obra.

\section{Bibliografía}

ALLENDE SALAZAR, J. (1925) Velázquez: des Meisters Gemäldes. Deutsche Verlags-Anstalt: Stuttgart-Berlin-Leipzig, 1925

BARDI, P. M. (1970) La obra pictórica completa de Diego Velázquez. Barcelona: Noguer-Rizzoli, 1970

BROWN, J. (1986) Velázquez: Painter and Courtier. New Haven and London: Yale University Press, 1986

BROWN, J.; GARRIDO M.C. (1998) Velázquez: The technique of Genius. New Haven: Yale University Press, 1998

CAMÓN AZNAR, J. (1964) Velázquez. Madrid: Espasa Calpe, 1964, 2 vol. CAMÓN AZNAR, J. (1978) La pintura española del siglo XVII. En Summa Artis. Madrid: Espasa-Calpe, 1978

COLECCIÓN Lázaro (1927). Expediente n 538, tomo II. Madrid, 1927 DURAN, A.; CASTAING, J.; WALTER, P. (2009) X-ray diffraction studies of Pompeian wall paintings using synchrotron radiation and dedicated laboratory made systems; Applied Physics A, article accepted DURAN, A.; PEREZ-RODRIGUEZ, J.L.; ESPEJO, T.; FRANQUELO, M.L.; CASTAING, J.; WALTER, P. (2009) Illuminated manuscripts characterization by laboratory-made portable XRD and micro-XRD systems. Analytical and Bioanalytical Chemistry, d.o.i. 10.1007/s00216-009-2992-5

FERNÁNDEZ, J. (2007-2008) Exposición-Catálogo. Teatro de la Grandeza (Andalucía Barroca). Granada

GÁLLEGO, J. (1974) Velázquez en Sevilla. Sevilla: Diputación Provincia GÁLLEGO, J. (1991) Velázquez (catálogo J. Gállego), cat. exp., Nueva York, Metropolitan Museum, 1989 y Madrid, Museo del Prado, 1990

GÁLLEGO, J. (1994) Velázquez en Sevilla. Sevilla: Diputación Provincial, 1994 GARRIDO PÉREZ, C. (1992) Velázquez: técnica y evolución. Madrid: Museo del Prado, 1992

GAYA NUÑO, J. A. (1958) La pintura española fuera de España. Historia y catálogo. Madrid: Espasa-Calpe, 1958

GIANNONCELLI, A.; CASTAING, J.; ORTEGA, L.; DOORYHÉE, E.; SALOMON, J.; WALTER, P.; HODEAU, J.L.; BORDET, P. (2008) A portable instrument for in situ determination of the chemical and phase compositions of cultural heritage objects. X-Ray Spectrometry, 37, 2008, pp. 418-423

GUDIOL RICART, J. (1973) Velázquez, 1599-1660. Barcelona: Ediciones Polígrafa, 1973

LÓPEZ REY, J. (1962) Velázquez. The artist as a Maker. Londres, 1962 LÓPEZ REY, J. (1963) Velázquez. A Catalogue Raisonné of His Oeuvre. London: Faber and Faber, 1963 LÓPEZ REY, J. (1996) Velázquez. Colonia: Taschen, 1996, 2 vol. LÓPEZ REY, J. (1998) Velázquez. La obra completa. Colonia: Taschen, Wildenstein Institute, 19982 vol.

MATTEINI, M. (2001) La química en la restauración: los materiales del arte pictórico. Sevilla: Nerea, Consejería de Cultura, Instituto Andaluz del Patrimonio Histórico, 2001

MAYER, A. L. (1936) Velázquez. A Catalogue Raisonné of the Pictures and Drawings. Londres, 1936

MENA, M. (1999) Catálogo de la Exposición Velázquez y Sevilla. Sevilla: Consejería de Cultura

NEWMAN, R. (1993) Observaciones acerca de los materiales pictóricos de Velázquez en Ciencia e Historia del Arte. Velázquez en el Prado. Madrid: Museo del Prado, 1993

PANTORBA, B. (1955) La vida y la obra de Velázquez. Madrid: Compañia Bibliográfica Española, 1955

PÉREZ SÁNCHEZ, A. E. (1992) Pintura Barroca Española (1600-1750). Madrid: Cátedra, 1992

PÉREZ SÁNCHEZ, A. E. (1995) Catálogo de la exposición Tres siglos de dibujo sevillano. Sevilla: FOCUS, 1995

PÉREZ SÁNCHEZ, A. E. (2005) Pintura española de los siglos XVII y XVIII en la Fundación Lázaro Galdiano. Madrid: Fundación Lázaro Galdiano, 2005 TORMO, E. (1923) Guias Regionales Calpe. Madrid: Espasa-Calpe, 1923 TRAPIER, E. (1948) Velázquez. Nueva York: Trustees, 1948 VON LOGA, V. (1913) Zur zeitbestimmung einiger werke des Velazquez. Jahrbuch der Königlich Preuszischen Kunstsammlungen 34 (1913), pp. 284-86, pl. 3 